\section{Has the rising incidence of Crohn's disease reached a plateau?}

Crohn's disease is a chronic condition of unknown cause and for which there is no effective medical treatment to prevent recurrence or spread. During 1934-77 the incidence of the disease in Cardiff greatly increased. ${ }^{1}$ Since Cardiff has a stable, clearly defined population and a good medical records system it is particularly suited for an epidemiological study. We have therefore examined the incidence of Crohn's disease during 1976-80 and related the findings to an earlier report. ${ }^{1}$

\section{Patients, methods, and results}

Patients who were resident in the City of Cardiff when newly diagnosed with Crohn's disease were identified from a diagnostic computer index and from personal records belonging to clinicians, radiologists, and pathologists. Patients were considered to have Crohn's disease only if $(a)$ the disease had been confirmed histologically in either the resected specimen or biopsy samples or $(b)$ there was radiological evidence of Crohn's disease and a suitable clinical history. The patient's age, date, and distribution of disease at the time of diagnosis as well as the method by which the diagnosis had been first made were noted. Details of the population structure of Cardiff were obtained from census data and used to calculate incidence figures.

The mean yearly incidence of Crohn's disease in Cardiff during 1976-80 was 4.92 cases 100000 population; this was not significantly greater than the $4 \cdot 83 / 100000$ during $1971-5$. The individual yearly incidences per 100000 during the five years were 3.2 in $1976,6.8$ in $1977,4.3$ in $1978,3.5$ in 1979 , and 6.7 in 1980 . The figures were also analysed according to sex and showed a slight preponderance of women $(1 \cdot 0: 1 \cdot 5)$. Among the 69 new cases, the distribution of disease at the time of diagnosis was ileocaecal in 35 , colonic in 21 , anorectal in five, small-bowel in three, and a combination of these sites in five. The initial diagnosis was made radiologically in 52 cases, by surgery with positive histological findings in 11 , and by sigmoidoscopy or colonoscopy with histological confirmation from biopsy in six.

When compared with the decade 1961-70 the number of new cases during 1971-80 had continued to rise, with an increase in all the major groups of Crohn's disease except anorectal disease (figure).

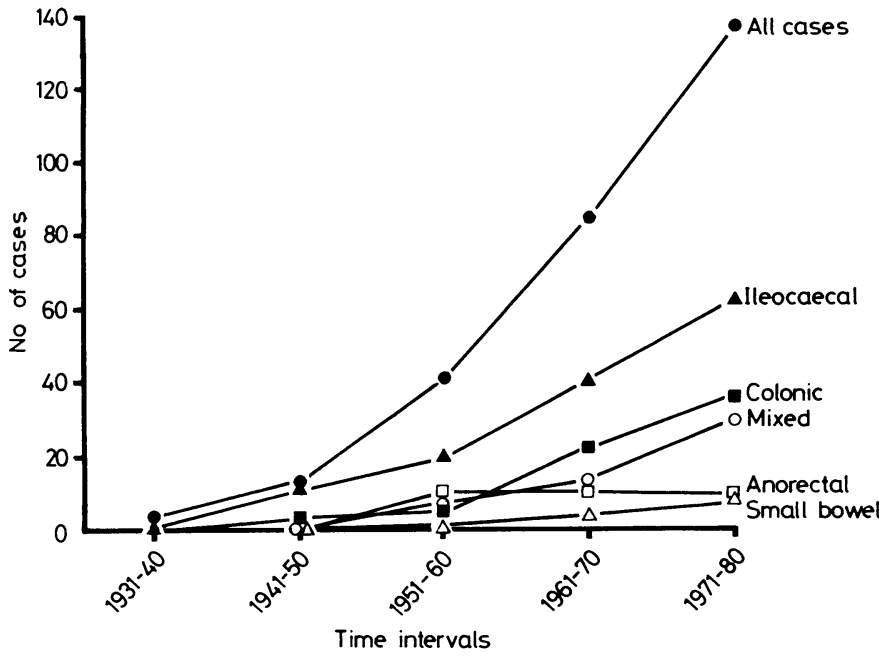

Number of new cases presenting during each decade from 1931 to 1980 : site of disease is that when first diagnosed.

\section{Comment}

Though there was a pronounced increase in new cases of Crohn's disease in Cardiff during 1971-80, the yearly incidence over the two five-year periods $1971-5$ and $1976-80$ had apparently levelled out at around 4.9 cases $/ 100000$ population. Though we do not attribute any importance to variations that occur from year to year, during 1977 and 1980 the incidence exceeded 6.5 cases $/ 100000$ population, which may suggest that the plateau is not yet stable. The highest recorded figures come from Malmö, Sweden, with an incidence of 6.0 cases/ 100000 population/year during $1966-73,{ }^{2}$ and figures from Cardiff continue to approach this.

Increased recognition of Crohn's colitis is not the main factor accounting for the rise, as all the major groups-particularly ileocaecal and mixed disease-are represented. This may partly be due to more frequent recognition of milder forms of the disease. Nevertheless, if this was so the standardised mortality rates for patients diagnosed in recent years would be expected to fall, which is not the case ${ }^{3}$; furthermore, deaths from Crohn's disease in England and Wales from 1950 to 1973 rose steadily. ${ }^{4}$ Since this condition places a heavy burden on medical, surgical, social, and community services, it is important to continue to monitor changes in the incidence.

We are grateful to the radiology department and colleagues who have helped to compile the list of new patients with Crohn's disease in Cardiff.

${ }^{1}$ Mayberry JF, Rhodes J, Hughes LE. Incidence of Crohn's disease in Cardiff between 1934 and 1977. Gut 1979:20:602-8.

2 Brahme F, Landstrom C, Wenckert A. Crohn's disease in a defined population. An epidemiological study of incidence, prevalence, mortality, and secular trends in the City of Malmö, Sweden. Gastroenterology 1975;69: 342-51.

${ }^{3}$ Mayberry JF, Newcombe RG, Rhodes J. Mortality in Crohn's disease. $Q \mathcal{F}$ Med 1980;49:63-8.

4 Office of Population Censuses and Surveys. Registrar General's statistical reviews of England and Wales for the years 1950-75. London: HMSO, 1951-73.

(Accepted 12 October 1981)

Department of Gastroenterology, University Hospital of Wales, Cardiff CF4 4XW

A D HARRIES, MA, MRCP, medical registrar

A BAIRD, medical student

J RHODES, MD, FRCP, consultant physician

Royal Gwent Hospital, Newport, Monmouthshire

J F MAYBERRY, MD, MRCP, medical registrar

\section{Discrepancy in results from three guaiacum resin tests}

The guaiacum resin test has been claimed to be an effective method of detecting occult blood loss due to asymptomatic cancer and precancerous lesions of the colon and rectum. ${ }^{1}$ Numerous proprietary versions of this test are readily available: the effectiveness of these products has been investigated, and the variation in results is by no means negligible. ${ }^{2}$ In the present study we compared the results obtained with three proprietary preparations for detecting occult blood in the faeces.

\section{Patients, methods, and results}

We studied 75 patients in hospital who had presented with symptoms suggestive of colorectal disease. The entire colon of each patient was examined by rectocolonoscopy.

The study compared the results obtained with HemoFEC, Haemoccult II, and Fecatest in patients maintained on a normal diet. The three tests were performed on the same stool sample before endoscopy and were interpreted blind within 12 hours, always by the same physician. Positive test results were considered to be falsely positive when no colorectal lesion was found on endoscopy.

The table shows the results. Overall, 33 patients had colonic lesions capable

Results obtained in 75 patients (33 presented with a colonic lesion)

\begin{tabular}{lccc}
\hline & HemoFEC & Haemoccult II & Fecatest \\
\hline Positive & 12 & 15 & 38 \\
Falsely positive & 2 & 4 & 18 \\
Negative & 63 & 60 & 37 \\
Falsely negative & 23 & 22 & 13 \\
\hline
\end{tabular}

of inducing occult blood loss: seven had cancer, 11 polyps, six ulcerative colitis, three Crohn's disease of the colon, three diverticulitis, and three other diseases. HemoFEC and Haemoccult II detected only three of the seven cancers and Fecatest five. HemoFEC and Haemoccult II detected only one and Fecatest four of the 11 cases of polyps. 


\section{Comment}

With all three tests for occult blood about $36 \%$ of the negative results obtained were falsely negative. This high proportion of falsenegative results is not surprising, since the tests were not used under optimal conditions ${ }^{3}$ : they were not repeated and no restrictions were placed on diet. A discrepancy in the results obtained with the three methods was noted. The Fecatest produced a large number of positive results, of which nearly half were falsely positive. Fecatest is highly sensitive, probably due to the method of preparing and preserving these test kits. ${ }^{4}$

Screening a large population for faecal occult blood using the above tests, especially Fecatest, would necessarily entail a costly and timeconsuming commitment to rectocolonoscopy or $x$-ray studies, or both owing to the excessive number of false-positive results obtained. This would therefore compromise the implied simplicity of a massive screening protocol and defeat the purpose of such testing.

${ }^{1}$ Greegor DH. Occult blood testing for detection of asymptomatic colon cancer. Cancer $1971 ; 28: 131-4$.

${ }^{2}$ Heinrich HC. Occult blood tests. Lancet $1980 ;$; :822-3.

3 Winawer SJ, Sherlock P, Schottenfeld D, Miller DG. Screening for colon cancer. Gastroenterology 1976;70:783-9.

${ }^{4}$ Adlercreutz H, Liewendahl K, Virkola P. Evaluation of Fecatest, a new guaiac test for occult blood in faeces. Clin Chem 1978;24:756-61.

(Accepted 7 October 1981)

Clinique des Maladies de l'Appareil Digestif, Hôpital Saint-Eloi, 34059 Montpellier, France

B FENEYROU, MD, interne des hôpitaux

$P$ BORIES, MD, assistant des hôpitaux

G POMIER-LAYRARGUES, MD, assistant des hôpitaux

H MICHEL, MD, professeur de clinique gastroenterologique

Service de Biochimie B, Hôpital Saint-Eloi

G GRAVAGNE, assistant de biologie des hôpitaux

\section{Multiple use of ordinary disposable syringes for insulin injections}

In a developing country such as Nigeria many insulin-dependent diabetics find the use of specially designed glass and plastic insulin syringes expensive and not easily available for purchase, unlike the ordinary multipurpose 2 or $2.5 \mathrm{ml}$ disposable plastic syringes. ${ }^{1}$ After finding that many diabetics were repeatedly using the same ordinary disposable syringe and needle because of difficulty in obtaining insulin syringes we encouraged them to continue with this practice and for many years have not observed any adverse effects. ${ }^{1}$ We undertook this study to find out whether this practice is indeed safe, bearing in mind that some Nigerian diabetics, particularly those from rural areas, do not have refrigerators for storing syringes and needles.

\section{Patients, methods, and results}

Twenty-one insulin-dependent Nigerian diabetics consented to take part in this study. Each was given either a 2 or $2.5 \mathrm{ml}$ ordinary disposable syringe and needle to be used repeatedly for their insulin injections. They continued with their usual dose of insulin (daily insulin zinc suspension lente) but were taught how to measure the dose in the new syringes. All were given 40 strength insulins (40 units). Each syringe had 10 markings per $\mathrm{ml}: 1 \mathrm{ml}$ of insulin in the syringe was equal to $\mathbf{4 0}$ units of insulin and each mark therefore equal to four units of insulin.

Patients were seen at weekly intervals for a month and thereafter monthly for six months and were told to bring their syringes, needles, and insulin to every clinic visit. At each visit the sites of the injections were inspected and the patient asked about any difficulties with the new technique. At three and six months samples were taken from the injection sites using premoistened swabs and cultured. Each swab was inoculated on to two blood agar plates, one being incubated aerobically and the other anaerobically for 24-48 hours at $37^{\circ} \mathrm{C}$. The swab was also inoculated on to Robertson's cooked-meat medium and incubated for 24 hours before subculturing on to two blood agar plates, which were then incubated aerobically and anaerobically. In addition, a small quantity of the insulin in use was sucked into the syringe being used at that time with the needle also in use and cultured by streaking on (i) two blood agar plates incubated aerobically and anaerobically for a minimum of seven days and inoculating on to (ii) brain-heart infusion broth and (iii) Robertson's cooked-meat broth. Incubation time for (ii) and (iii) was seven days at $37^{\circ} \mathrm{C}$ before subcultures were made on to blood agar plates, which were then incubated aerobically and anaerobically for $\mathbf{4 8}$ hours.

When the syringe became disfigured or the markings started to disappear it was changed. The needles were changed as often as they became blunt For home storage patients were told that after each injection they should carefully recap the needle and place the syringe with needle attached into its packet and then store it dry with the bottle of insulin in a clean container covered with a lid. The patients were asked to report immediately if any tenderness, swelling, or redness occurred at the site of injections.

Duration of use of a syringe varied from seven to 49 days, and for a needle from three to nine days. The total number of insulin injections in this study was 1890 . The table shows the average durations of use of the syringes and needles.

All patients expressed general satisfaction with the new technique. No deterioration in diabetic control was noted. Only one patient complained of soreness at the site of injection, but no local inflammation was seen in any patient. Skin swabs taken from the injection sites yielded no pathogens except on one occasion, when a heavy growth of Staphylococcus aureus was isolated from a patient who had an infection of the pulp of one of his fingers, caused by this organism. On three occasions normal flora-namely, Staph epidermidis-was recovered. Culture of the insulin solutions used yielded no pathogens.

Average duration of use (number of days) of disposable syringes and needles

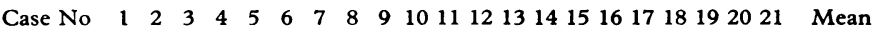
\begin{tabular}{lllllllllllllllllllllllllll}
\hline Syringes & 9 & 22 & 30 & 28 & 15 & 21 & 29 & 38 & 42 & 18 & 45 & 36 & 29 & 16 & 22 & 30 & 34 & 14 & 19 & 32 & 26 & $26 \cdot 4$ \\
Needles & 4 & 5 & 4 & 6 & 6 & 4 & 5 & 6 & 8 & 3 & 8 & 7 & 6 & 6 & 5 & 8 & 4 & 5 & 6 & 8 & 5 & $5 \cdot 7$
\end{tabular}

\section{Comment}

Some authors ${ }^{2} 3$ have found no bacteriological risk in the repeated use of plastic disposable insulin syringes and needles stored in refrigerators. We found that, although refrigerators were not used for storage, the cheap ordinary 2 or $2.5 \mathrm{ml}$ disposable syringes can be safely used repeatedly by the same person without adverse reactions or risk of infection. Provided the syringes and needles are stored dry the risk of infections, particularly with mycobacteria, ${ }^{4}$ appears low. Furthermore, the additives $(0 \cdot 15 \%$ metacresol and $0.06 \%$ phenol) to insulin may inhibit up to $10^{8}$ colony-forming units of Staph aureus, even when incubated at room temperature. ${ }^{3}$ This value greatly exceeds what occurs naturally as contamination, even with uncleansed human skin. Measuring an insulin dose with the 2 or $2.5 \mathrm{ml}$ ordinary syringe is simple provided there are up to 10 marks in a millilitre and insulin strengths of $40 \mathrm{U}$ are used. With higher strengths finer adjustments of dose may be difficult.

Variable deterioration ( $82-97 \%$ of normal) in potency has been found in insulin kept for three months at $36^{\circ} \mathrm{C} . .^{5}$ We therefore usually prescribe not more than two months' supply of insulin at a time, thus minimising deterioration.

We must emphasise that the technique used in this study is to be recommended only to diabetics who cannot afford or be provided with the usual insulin syringes and needles, and who have no refrigerators for storage at their disposal. Such situations are usually found in developing countries, particularly in the rural areas. From this study the bacteriological risk of this technique appears very small.

We thank Miss Comfort Ogbogu for secretarial help.

1 Oli JM. Disposable syringes for insulin injection. Br Med $\mathcal{f}$ 1979;ii:273. 2 Greenough A, Cockcroft PM, Bloom A. Disposable syringes for insulin injection. $\mathrm{Br} \mathrm{Med} \mathcal{F} 1979 ; \mathrm{i}: 1467-8$

${ }^{3}$ Hodge RH Jr, Krongaard L, Sande MA, Kaiser DL. Multiple use of disposable insulin syringe-needle units. FAMA 1980;244:266-7.

4 Jackson PG, Keen H, Noble CJ, Simmons NA. Injection abscesses in a diabetic due to Mycobacterium chelonei var abscessus. $\mathrm{Br} \mathrm{Med} \mathcal{F}$ $1980 ; 281: 1105-6$.

5 Stephenson NR, Romans RG. Thermal stability of insulin made from zinc insulin crystals. F Pharm Pharmacol 1960;12:372-3.

(Accepted 15 September 1981)

Departments of Medicine and Microbiology, University of Nigeria Teaching Hospital, Enugu, Nigeria

J M OLI, MRCP, FMCP, senior lecturer

H C GUGNANI, PHD, professor of microbiology

G C OJIEGBE, FIMLT, technologist 